\title{
g-factor and exchange energy in a few-electron lateral InGaAs quantum dot
}

M. Larsson, H. A. Nilsson, H. Hardtdegen, and H. Q. Xu

Citation: Appl. Phys. Lett. 95, 192112 (2009);

View online: https://doi.org/10.1063/1.3264053

View Table of Contents: http://aip.scitation.org/toc/apl/95/19

Published by the American Institute of Physics

\section{Articles you may be interested in}

Top-gate defined double quantum dots in InAs nanowires Applied Physics Letters 89, 252106 (2006); 10.1063/1.2409625

Measurements of the spin-orbit interaction and Landé g factor in a pure-phase InAs nanowire double quantum dot in the Pauli spin-blockade regime

Applied Physics Letters 109, 053106 (2016); 10.1063/1.4960464

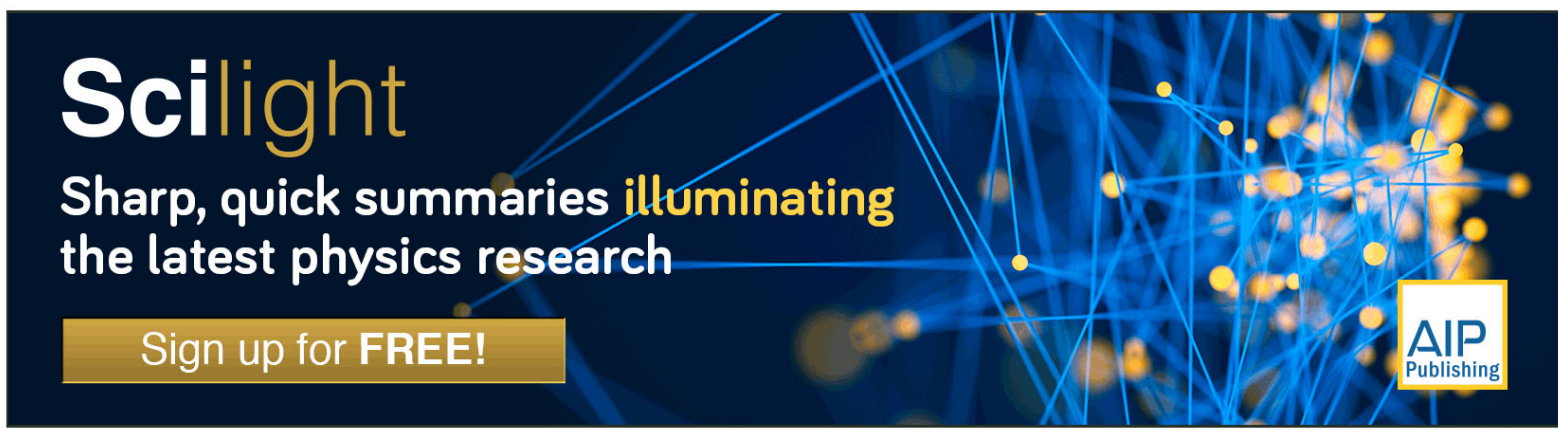




\title{
g-factor and exchange energy in a few-electron lateral InGaAs quantum dot
}

\author{
M. Larsson, ${ }^{1}$ H. A. Nilsson, ${ }^{1}$ H. Hardtdegen, ${ }^{2}$ and H. Q. Xu ${ }^{1, a)}$ \\ ${ }^{1}$ Division of Solid State Physics, Lund University, P.O. Box 118, S-221 00 Lund, Sweden \\ ${ }^{2}$ Institute of Bio- and Nanosystems (IBN-1), Juelich Aachen Research Alliance (JARA), Research Center \\ Juelich, D-52425 Juelich, Germany
}

(Received 17 September 2009; accepted 25 October 2009; published online 13 November 2009)

\begin{abstract}
We report on the measurements of the g-factor and the exchange interaction of electrons in a few-electron lateral quantum dot formed in an InGaAs/InP semiconductor heterostructure. The spin filling sequence of the electron states in the dot is determined by magnetotransport measurements and parallel spin filling configurations are identified. The measured g-factor (for a magnetic field applied parallel to the InGaAs quantum-well layer) has a value in the range of $\left|g^{*}\right| \approx 2$ to $\left|g^{*}\right| \approx 4$ and is strongly level-dependent. By analysis of the energies of the states which favor a parallel spin filling, the lower bound of the exchange energy of electrons in the dot in the order of $\sim 210 \mu \mathrm{eV}$ is extracted. (C) 2009 American Institute of Physics. [doi:10.1063/1.3264053]
\end{abstract}

Quantum dots made from semiconductor materials have been extensively investigated in recent years due to an increasing interest in the studies of the fundamental physics revealed in such artificial atomic systems and in the developments of semiconductor based spintronic devices and solid state based devices for quantum information processing and communication. ${ }^{1-4}$ In these studies and developments, knowledge of the g-factor, spin-orbit interaction, and exchange energy, e.g., the difference in energy between a singlet and triplet ground state, of electrons and/or holes is of great importance. ${ }^{5-7}$ Quantum devices made from InGaAs/ InP heterostructures are of particular interest because of the existence of a high effective electron g-factor ${ }^{8-10}$ and strong, tunable spin-orbit interaction in the InGaAs quantum well material. ${ }^{11}$ However, studies of planar InGaAs quantum dot devices defined in InGaAs/InP heterostructures have been hindered by difficulties in device fabrication due to a low Schottky barrier formed in the interface between a metal and the heterostructure surface ${ }^{12}$ and only very recently successful realizations of such InGaAs quantum dots in the manyelectron regime have been reported. ${ }^{13,10}$

Here we report on realization of a few-electron quantum dot in an InGaAs/InP heterostructure by employing the technologies of local finger gating and wet chemical etching. By studying the ground state energy levels of the few-electron quantum dot in the Coulomb blockade regime as a function of a magnetic field applied parallel to the InGaAs quantum well layer, the g-factor and the spin-filling sequence of electrons in the dot were determined. We observed that there exists a group of ground state energy levels which show a parallel spin filling sequence. The results can be understood using a simple model in which the exchange energy of electrons with parallel spins in the dot is taken into account.

The device studied in this work was fabricated from a modulation-doped InGaAs/InP heterostructure. The material was grown by metal-organic vapor phase epitaxy on a semiinsulating InP substrate. A $50 \mathrm{~nm}$ thick InP buffer was grown on top of the semi-insulating InP substrate, followed by a $9 \mathrm{~nm}$ thick $\operatorname{In}_{0.75} \mathrm{Ga}_{0.25}$ As quantum-well layer, a $20 \mathrm{~nm}$ thick

\footnotetext{
${ }^{\text {a) }}$ Author to whom correspondence should be addressed. Electronic mail: hongqi.xu@ftf.lth.se.
}

InP spacer, a $1 \mathrm{~nm}$ thick Si-doped $\mathrm{InP}$ layer, and finally a $20 \mathrm{~nm}$ thick InP cap layer. A two-dimensional electron gas (2DEG) was formed in the InGaAs quantum well layer. Hall measurements performed at a temperature of $300 \mathrm{mK}$ showed a mobility and a sheet electron concentration of $1.0 \times 10^{5} \mathrm{~cm}^{2} \mathrm{~V}^{-1} \mathrm{~s}^{-1}$ and $7.5 \times 10^{11} \mathrm{~cm}^{-2}$ in the 2DEG, respectively. To fabricate the lateral InGaAs quantum dot device, we first defined a lateral quantum wire in the 2DEG by electron beam lithography (EBL) and wet chemical etching. A 150-200 nm thick gate dielectric, made of cross-linked polymethylmethacrylate (PMMA), was then deposited on top of the quantum wire. Finally five local finger Ti/Au gates were fabricated on top of the gate dielectric, covering the quantum wire, by EBL and thermal evaporation of metal. The width and length of the quantum wire were 300 and $800 \mathrm{~nm}$, respectively. The width of the finger gates was about $40 \mathrm{~nm}$ and the period of the finger gates was $100 \mathrm{~nm}$. Figure 1(a) shows a scanning electron microscope (SEM) image of

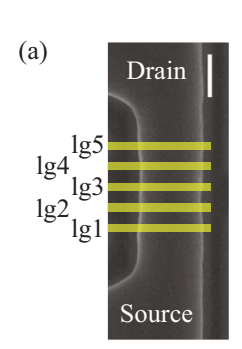

(c)

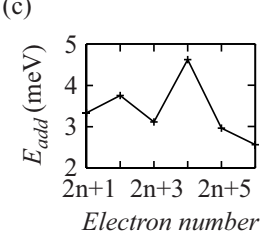

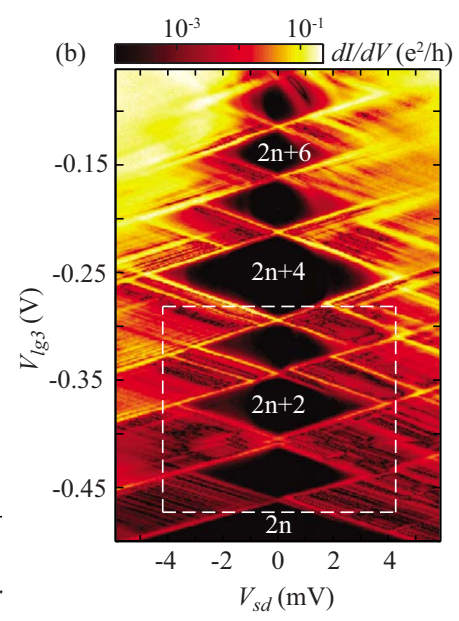

FIG. 1. (Color online) (a) SEM image of a quantum wire structure defined by etching trenches before deposition of the gate dielectric. The local finger gates are added schematically as yellow lines for device illustration. The scale bar is $200 \mathrm{~nm}$. (b) Differential conductance as a function of sourcedrain bias $V_{s d}$ and gate voltage $V_{\lg 3}$, plotted on a logarithmic color scale, showing Coulomb blockade diamonds typical for the electron transport in a few-electron quantum dot. A parametric charge rearrangement can be seen at $V_{\lg 3}=-0.41 \mathrm{~V}$. (c) Addition energy as a function of the electron number in the dot, showing a typical shell filling behavior. 
an etched quantum wire structure before deposition of PMMA with the five local finger gates added schematically as yellow lines for illustration.

Transport measurements of the device were performed in a dilution refrigerator at a temperature of about $100 \mathrm{mK}$ with a dc bias applied symmetrically to the source and drain contacts. Negative dc voltages were applied to the local finger gates to induce tunable electrostatic barriers along the quantum wire and, thereby, form a single or a double quantum dot in the quantum wire. In this letter, we will only consider transport measurements of a single quantum dot formed by applying negative voltages to local finger gates $\lg 2$ and $\lg 4$. The pinch-off voltages were about -4.1 and $-4.8 \mathrm{~V}$ for local finger gates $\lg 2$ and $\lg 4$. For the measurements presented in this work, the voltages applied to the two local finger gates were $V_{\mathrm{lg} 2}=-3.6 \mathrm{~V}$ and $V_{\mathrm{lg} 4}=-4.2 \mathrm{~V}$. The electron occupation in the dot was tuned by a voltage applied to finger gate $\lg 3$. Figure 1(b) shows the measured differential conductance of the dot as a function of source-drain bias $V_{s d}$ and gate voltage $V_{\lg 3}$ (charge stability diagram) at zero magnetic field. Diamond-shaped regions of low conductance are clearly seen in the figure, which is typical for electron transport through a quantum dot in the Coulomb blockade regime. An average voltage to energy conversion factor of $\alpha=0.056$ for $V_{\lg 3}$ was determined by looking at the slopes of the diamond borders of Fig. 1(b). Figure 1(b) also shows a clear even-odd electron-occupation-number behavior in the addition energy of electrons on the dot, see also Fig. 1(c). This behavior of the addition energy stems from spin degeneracy of the energy levels and is a signature of a quantum dot in the few-electron regime. ${ }^{14}$ The addition energies shown in Fig. 1(c) are in the ranges of $2-5 \mathrm{meV}$, which are considerably larger than the addition energies of about $1 \mathrm{meV}$ seen in quantum dots in the many-electron regime made from the same material system. ${ }^{13,10}$ Although the dot studied here is in the few-electron regime we were unable to completely empty the dot of electrons. Cotunneling through the dot leading to an increase in the conductance in the Coulomb blockaded regions ${ }^{15}$ can also be seen inside the Coulomb diamonds in Fig. 1(b).

In order to determine the spin filling sequence and the electron effective g-factor $g_{n}^{*}$ of the quantum levels in the few-electron quantum dot, we measured the evolution of the conductance peaks in the linear response regime with increasing magnetic field applied parallel to the quantum well layer. Figure 2(a) shows the results of such measurements for the quantum dot at $V_{s d}=25 \mu \mathrm{V}$. In a typical spin filling sequence with alternating fillings of spin- $\uparrow$ and spin- $\downarrow$ electrons, one expects that the two conductance peaks located just above and below an odd-number electron Coulomb diamond is going to be split with increasing magnetic field according to $\Delta E(B)=\Delta E(0)+\left|g_{n}^{*} \mu_{B} B\right|$ (the Zeeman effect), where $n$ is the single-particle level index which can be associated with the number of electrons in the dot $N$ as $n=(N$ $+1) / 2$. For an even-number electron Coulomb diamond, the energy difference of the two corresponding conductance peaks is developed according to $\Delta E(B)=\Delta E(0)-\left|g_{n}^{*} \mu_{B} B\right| / 2$ $-\left|g_{n-1}^{*} \mu_{B} B\right| / 2$, where the single-particle level index $n$ is then associated with the number of electrons in the $\operatorname{dot} N$ as $n$ $=N / 2$. Thus, the two associated conductance peaks will move closer as the magnetic field is increased. However, for the four measured conductance peaks shown in the lower

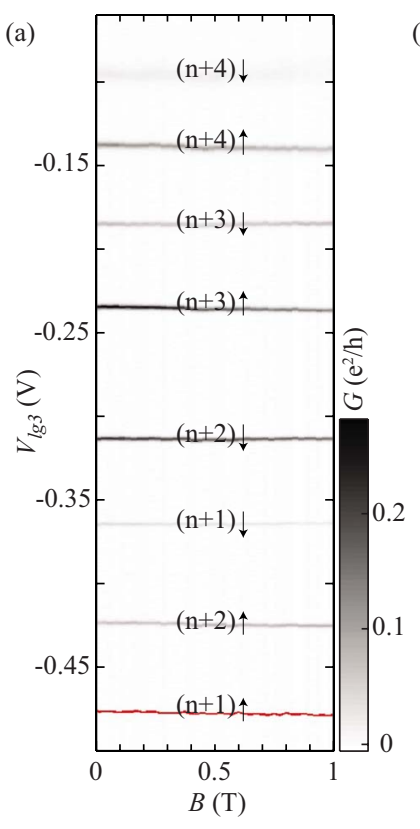

(c)

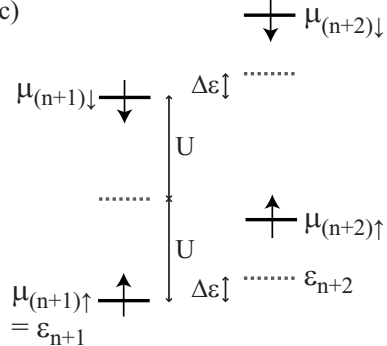

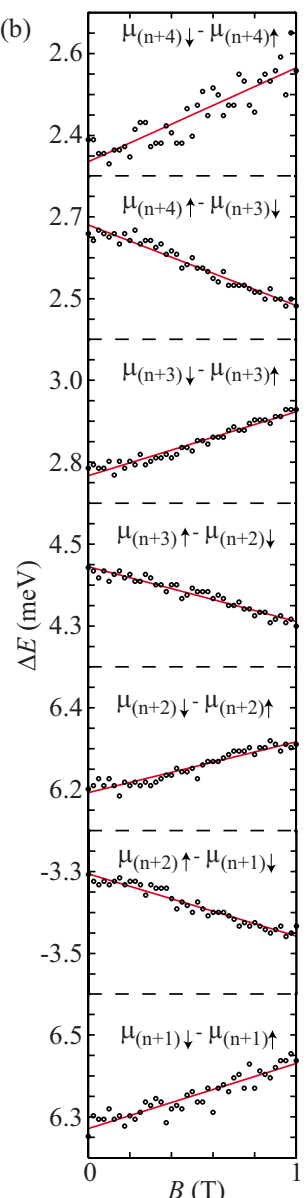

FIG. 2. (Color online) (a) Conductance of the quantum dot measured in the linear response regime as a function of the magnetic field applied parallel to the quantum well layer. The numbers in parentheses and arrows indicate the level indices of the last filled single-particle levels and spin states of the levels. Here negative values of the g-factors are assumed. (b) Magnetic field evolution of the energy difference between the measured conductance peaks shown in (a). In each subplot, the orbital level and spin indices of the two associated states are indicated. (c) A simple model showing the four energy states of the dot studied in the region marked by the dashed box in Fig. 1(b) Here it is assumed that the two single-particle orbitals $\epsilon_{n+1}$ and $\epsilon_{n+2}$ are nearly degenerate and the negative exchange energy of the electrons favors a parallel spin filling.

part of Fig. 2(a), the magnetic field evolutions of the energy differences between neighboring peaks do not show the characteristics described just above. The reason is that here the two involved single-particle orbital levels are degenerate or nearly degenerate and the exchange interaction between electrons favors a parallel spin filling instead of an alternating spin filling commonly observed in a quantum dot without encountering degenerate or nearly degenerate single-particle orbital levels. Thus, by taking into account the single-particle orbital level degeneracy, or near single-particle orbital level degeneracy, and the (negative) exchange energy between electrons of parallel spins, the spin filling sequence of the electrons in the dot in the linear response regime is determined to be $(n+1) \uparrow,(n+2) \uparrow,(n+1) \downarrow,(n+2) \downarrow,(n+3) \uparrow,(n$ $+3) \downarrow,(n+4) \uparrow$, and $(n+4) \downarrow$, accounting from the lowest lying state to the highest lying one in Figs. 1(b) and 2(a). It should be noted that degenerate or nearly degenerate orbital levels can occur due to the lateral shape of the dot even though the dot is in the few-electron regime. Additionally, if we assume nondegenerate orbital levels with a spin filling sequence al- 
ternating between spin- $\uparrow$ and spin- $\downarrow$ electrons we see a magnetic field evolution of the energy differences between neighboring peaks which is contradictory to the assumption.

Figure 2(b) shows the magnetic field evolution of the energy differences between conductance peaks corresponding to the states indicated by the quantum level index and spin arrow in each subplot. Clearly, it is seen that the energy difference between the two opposite spin states with the same orbital index increases with increasing magnetic field, while the energy difference between two opposite spin states which belong to two neighboring orbital levels decreases with increasing magnetic field. Note that the energy difference between the spin- $\uparrow$ state of the $n+2$ level and the spin- $\downarrow$ state of the $n+1$ level, $\mu_{(n+2) \uparrow}-\mu_{(n+1) \downarrow}$, is negative, because, as a result of the parallel spin filling, the former state is located below the latter one. By linear fits to the data points in Fig. 2(b) the effective g-factors of the measured singleparticle states can be determined. The results are $\left|g_{n+1}^{*}\right|=2.7$, $\left|g_{n+2}^{*}\right|=2.1,\left|g_{n+3}^{*}\right|=2.7$, and $\left|g_{n+4}^{*}\right|=4.0$. The g-factors found in our few-electron dot are slightly larger than the g-factor found previously for a many-electron dot made from the same material system ${ }^{10}$ and than the g-factor of InGaAs/InP quantum wells determined using the optically detected magnetic resonance technique. ${ }^{8}$ The difference in the g-factor between the few- and many-electron dot cases as well as the level-to-level fluctuations of the g-factor in the dot can be attributed to variations of the spin-orbit interaction strength present in the dot. ${ }^{16,17}$

We now focus on the two degenerate or nearly degenerate orbital states with parallel spin filling and analyze the exchange interaction of electrons in the dot. With the use of the schematic for the four energy states shown in Fig. 2(c) and the assumption of a constant charging energy $U$, the energy differences between the four states can be written as

$$
\begin{aligned}
& \mu_{(n+1) \downarrow}-\mu_{(n+1) \uparrow}=2 U, \\
& \mu_{(n+2) \uparrow}-\mu_{(n+1) \uparrow}=\Delta \epsilon+U-\left|\epsilon_{x, \uparrow \uparrow}\right|, \\
& \mu_{(n+2) \downarrow}-\mu_{(n+1) \downarrow}=\Delta \epsilon+U-\left|\epsilon_{x, \downarrow \downarrow}\right|,
\end{aligned}
$$

where $\Delta \epsilon=\epsilon_{n+2}-\epsilon_{n+1} \geq 0$ is the single-particle level spacing, and $\epsilon_{x, \uparrow \uparrow}$ and $\epsilon_{x, \downarrow \downarrow}$ are the exchange energy of the two spin- $\uparrow$ electrons and the exchange energy of the two spin- $\downarrow$ electrons, respectively. Based on Eq. (1), a constant charging energy of $U=3.54 \mathrm{meV}$ can be determined using the first two addition energies given in Fig. 1(c). The exchange energies $\left|\epsilon_{x, \uparrow \uparrow}\right|$ and $\left|\epsilon_{x, \downarrow \downarrow}\right|$ can then be extracted from the corresponding addition energies in Fig. 1(c) using Eqs. (2) and (3). However, we have been unable to determine the exact single-particle level spacing $\Delta \epsilon$ and are, therefore, only able to determine the quantities of $\left|\epsilon_{x, \uparrow \uparrow}\right|=210 \mu \mathrm{eV}+\Delta \epsilon$ and $\left|\epsilon_{x, \downarrow \downarrow}\right|=430 \mu \mathrm{eV}+\Delta \epsilon$, which give the lower bounds of the exchange energies since $\Delta \epsilon \geq 0$. Assuming a single-particle level spacing of a few hundred $\mu \mathrm{eV}$, the values extracted for the exchange energy in the studied few-electron lateral $\mathrm{In}_{0.75} \mathrm{Ga}_{0.25} \mathrm{As}$ quantum dot correspond well with the values determined for a vertical $\operatorname{In}_{0.05} \mathrm{Ga}_{0.95}$ As quantum dot in the few-electron regime. ${ }^{5}$ It should be noted that the exchange energy extracted from our measurements using our simple model increases with electron occupation, which is in contrast to the results of Ref. 5, where a decrease in the exchange energy with electron occupation was observed. The increase in the exchange energy with electron number could partially be explained by the fact that an average charging energy was used in our analysis, which will result in an underestimation of $\left|\epsilon_{x, \uparrow \uparrow}\right|$ and an overestimation of $\left|\epsilon_{x, \downarrow \downarrow}\right|$ since the charging energy decreases with electron occupation in the dot.

In summary, we have performed magnetotransport measurements of a few-electron quantum dot formed in an InGaAs/InP semiconductor heterostructure. The spin filling sequence and the g-factors of a few low energy levels were determined by studying the evolution of the conductance peaks with a magnetic field in the linear response regime. The measured g-factors were in the range of $\left|g^{*}\right| \approx 2$ to $\left|g^{*}\right|$ $\approx 4$ and were strongly level dependent. Parallel spin filling configurations were observed and the corresponding orbital degeneracy or near degeneracy was identified. The results were interpreted as a consequence of the exchange interaction of electrons with parallel spins in the dot. Based on a simple, constant charging energy model, the exchange energy was extracted to be in the order of $\geq 210 \mu \mathrm{eV}$ in the dot.

This work was supported by the Swedish Research Council (VR) and by EU program SUBTLE (EU Contract No. 034236).

${ }^{1}$ R. Hanson, L. P. Kouwenhoven, J. R. Petta, S. Tarucha, and L. M. K. Vandersypen, Rev. Mod. Phys. 79, 1217 (2007).

${ }^{2}$ D. J. Reilly, J. M. Taylor, J. R. Petta, C. M. Marcus, M. P. Hanson, and A. C. Gossard, Science 321, 817 (2008).

${ }^{3}$ F. H. L. Koppens, C. Buizert, K. J. Tielrooij, I. T. Vink, K. C. Nowack, T. Meunier, L. P. Kouwenhoven, and L. M. K. Vandersypen, Nature (London) 442, 766 (2006).

${ }^{4}$ J. R. Petta, A. C. Johnson, J. M. Taylor, E. A. Laird, A. Yacoby, M. D. Lukin, C. M. Marcus, M. P. Hanson, and A. C. Gossard, Science 309, $2180(2005)$

${ }^{5}$ S. Tarucha, D. G. Austing, Y. Tokura, W. G. van der Wiel, and L. P. Kouwenhoven, Phys. Rev. Lett. 84, 2485 (2000).

${ }^{6}$ A. Fuhrer, T. Ihn, K. Ensslin, W. Wegscheider, and M. Bichler, Phys. Rev. Lett. 91, 206802 (2003).

${ }^{7}$ S. Roddaro, A. Fuhrer, P. Brusheim, C. Fasth, H. Q. Xu, L. Samuelson, J. Xiang, and C. M. Lieber, Phys. Rev. Lett. 101, 186802 (2008).

${ }^{8}$ B. Kowalski, P. Omling, B. K. Meyer, D. M. Hofmann, C. Wetzel, V. Härle, F. Scholz, and P. Sobkowicz, Phys. Rev. B 49, 14786 (1994).

${ }^{9}$ T. Schäpers, V. A. Guzenko, and H. Hardtdegen, Appl. Phys. Lett. 90, 122107 (2007).

${ }^{10}$ J. Sun, M. Larsson, I. Maximov, H. Hardtdegen, and H. Q. Xu, Appl. Phys. Lett. 94, 042114 (2009).

${ }^{11}$ T. Koga, J. Nitta, T. Akazaki, and H. Takayanagi, Phys. Rev. Lett. 89, 046801 (2002).

${ }^{12}$ H. Cetin and E. Ayyildiz, Semicond. Sci. Technol. 20, 625 (2005).

${ }^{13}$ M. Larsson, D. Wallin, and H. Q. Xu, J. Appl. Phys. 103, 086101 (2008)

${ }^{14}$ S. Tarucha, D. G. Austing, T. Honda, R. J. van der Hage, and L. P. Kouwenhoven, Phys. Rev. Lett. 77, 3613 (1996).

${ }^{15}$ S. De Franceschi, S. Sasaki, J. M. Elzerman, W. G. van der Wiel, S Tarucha, and L. P. Kouwenhoven, Phys. Rev. Lett. 86, 878 (2001).

${ }^{16}$ S. Csonka, L. Hofstetter, F. Freitag, S. Oberholzer, C. Schönenberger, T. S. Jespersen, M. Aagesen, and J. Nygård, Nano Lett. 8, 3932 (2008)

${ }^{17}$ H. A. Nilsson, P. Caroff, C. Thelander, M. Larsson, J. B. Wagner, L.-E. Wernersson, L. Samuelson, and H. Q. Xu, Nano Lett. 9, 3151 (2009). 